\title{
Colletotrichum asianum causes anthracnose in Philippine mango cv. Carabao
}

\author{
Lourdes V. Alvarez ${ }^{1} \cdot$ Yukako Hattori $^{2} \cdot$ Chester C. Deocaris $^{1} \cdot$ Carmelita P. Mapanao $^{1} \cdot$ Arcibel B. Bautista $^{1}$. \\ Mark Joseph B. Cano ${ }^{1} \cdot$ Kouske Naito $^{2} \cdot$ Shunsuke Kitabata $^{2} \cdot$ Keiichi Motohashi $^{3} \cdot$ Chiharu Nakashima $^{2}$
}

Received: 13 May 2019 / Accepted: 2 March 2020 / Published online: 12 March 2020

(C) Australasian Plant Pathology Society Inc. 2020

\begin{abstract}
Anthracnose symptoms were observed on fruits and leaves of mango cv. Carabao at several locations in the Philippines. Single spore isolates were generated from affected leaves and fruits and identified as Colletotrichum asianum based on morphology and molecular analyses using several genes. Inoculation of $C$. asianum isolates onto healthy fruit of mango cv. Carabao resulted in the same symptoms as those observed initially. Koch's postulates were satisfied after isolates made from symptomatic inoculated fruits were morphologically identical as the original isolates. This is the first proof of $C$. asianum as causal fungus of anthracnose in mango cv. Carabao in the Philippines.
\end{abstract}

Keywords Anthracnose $\cdot$ Mangifera indica $\cdot$ Colletotrichum asianum $\cdot$ Colletotrichum gloeosporioides

The mango (Mangifera indica: Anacardiaceae) is one of the most popular fruits grown in the tropical and subtropical zones of the world (Alemu et al. 2014). Mango cv. Carabao is considered as premium agricultural export in the Philippines (Simeon 2017) being the third most important fruit crop based on export volume and value (Department of AgricultureBureau of Plant Industry 2016). Production of mango cv. Carabao dropped by $3.3 \%$ from 546,000 tons in the 3rd quarter of 2017 to 528,000 tons over the same period in 2018 (PSA 2018). Philippine Statistics Authority attributed the low production to several factors such as occurrence of rainfall, adverse effect of insect pests, particularly cecid fly, and fungal diseases.

There are many diseases of mango worldwide (Alemu 2014; Prakash and Misra 2000). Among them, anthracnose caused by C. gloeosporioides, is reported as one of the most important fungal disease and is the major pre- and postharvest disease of mango in the Philippines and throughout

Lourdes V. Alvarez

lvalvarez@pup.edu.ph

1 College of Science, Polytechnic University of the Philippines, Sta. Mesa, Manila, Philippines

2 Graduate school of Bioresources, Mie University, Kurima-Machiya 1577, Tsu, Mie 514-8507, Japan

3 Tokyo University of Agriculture, Sakuraoka 1-1-1, Setagaya, Tokyo 156-8502, Japan the tropics (Dodd et al. 1991; Jeffries et al. 1990). Throughout the years, $C$. gloeosporioides was reported to be the causative agent of anthracnose in mango cv. Carabao in the Philippines where most of the studies conducted focused more on the disease control (Alvindia and Acda 2015; Esguerra et al. 2006; Lizada et al. 1986). Weir et al. (2012) verified genetically by multi-gene phylogenies that $C$. gloeosporioides is a species complex which consisted of 22 taxa plus one subspecies. It is therefore possible that the anthracnose-causing fungi of mango cv. Carabao could be any of the 22 taxa or subspecies under the $C$. gloeosporioides species complex. The present study investigated the identity of the fungus causing anthracnose disease of Philippine mango cv. Carabao at several localities in the Philippines, using morphology, molecular analyses and pathogenicity studies.

In 2017, anthracnose-symptomatic fruit and leaf samples of mango cv. Carabao were collected through opportunistic sampling from a mango orchard in the province of Iba, Zambales in Luzon region and two orchards in Jordan, Guimaras in Visayas region. A total of 28 samples were collected in Zambales and 46 samples in Guimaras. The symptoms in leaves were irregularly shaped, brown to black spots and on fruit sunken, prominent, black necrotic spots. Yellow-orange acervuli were often present within the spots and no setae were present in the acervuli (Fig.1). A single conidium isolates of the fungus were generated using the methods described in Nakashima et al. (2016). A mass of conidia from a single acervulus was suspended in a drop of sterile distilled water and the suspension was streaked across the surface of water 
Fig. 1 Anthracnose symptoms on mango cv. Carabao: Necrotic spots (A) and coalesced lesions (B)

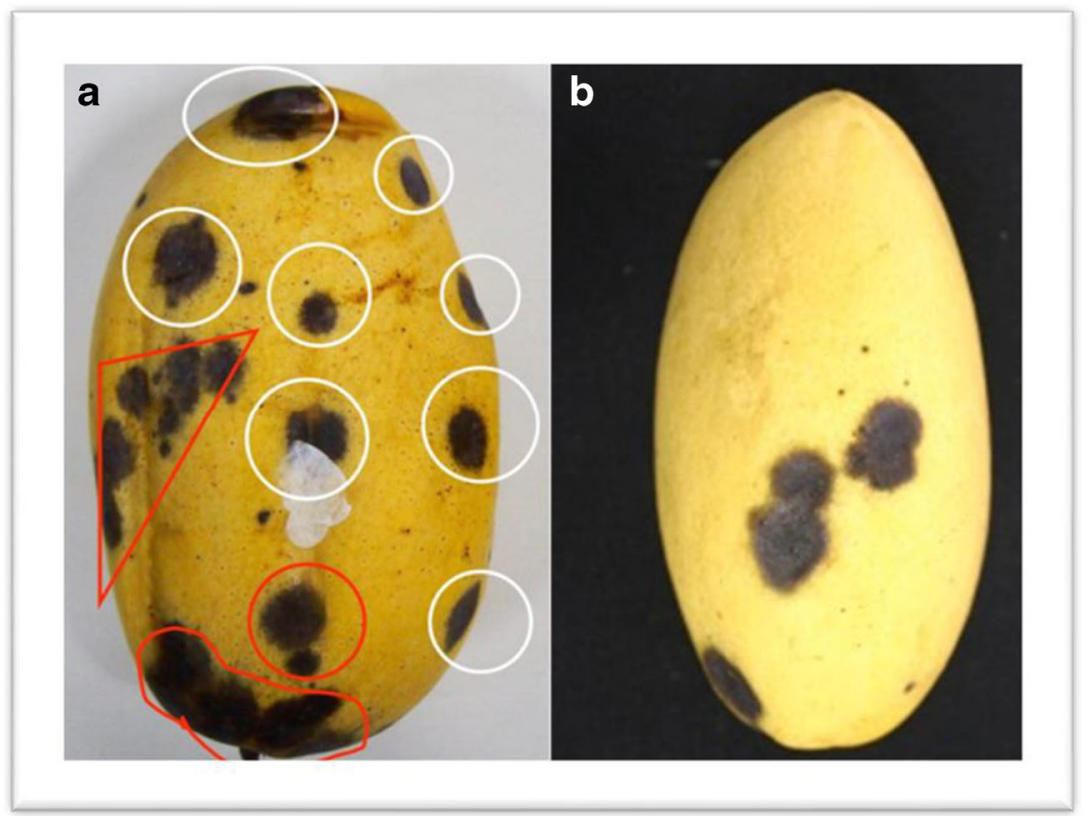

agar in 9-cm-diameter petri dishes which were incubated for $24 \mathrm{~h}$ at room temperature $\left(25^{\circ}-30^{\circ} \mathrm{C}\right)$ under ambient light. Single germinating conidium was selected with the aid of a compound microscope and a circle of agar surrounding the conidium was excised using a modified loop and transferred to potato dextrose agar (PDA; Nissui) in similar petri dishes. The cultures were incubated at room temperature $\left(25^{\circ}-30^{\circ} \mathrm{C}\right)$ under ambient light. Single conidium isolates were also grown on synthetic nutrient-poor agar (SNA) under the same conditions to determine the morphology of appressoria and conidiogenous cells. Three isolates were subjected for further analyses PHP2017_Z23, PHP2017_Z25 and PHP2017_Z26. The isolates were deposited at the University of the Philippines Culture Collection (UPCC) housed at the Natural Science Research Institute (NSRI) with the Accession Numbers: UPCC 4139, UPCC 4138 and UPCC 4137, respectively.
Colonies of the three Colletotrichum isolates on PDA were white, later grayish green to black, white at the edge, with a mean colony diameter of $71.33 \pm 2.1 \mathrm{~mm}(n=3)$ from a single conidium after 10 days at $25^{\circ} \mathrm{C}$ under ambient light; aerial mycelium was cottony, white, often formed concentric rings; acervuli were blackish with orange conidial masses scattered or formed concentric rings on the surface of the colony; reverse initially light orange and later black starting from the middle, (Fig.2). Phialides were hyaline, 11-28 × 2-4.5 $\mu \mathrm{m}$ and conidia were aseptate, hyaline, cylindrical to fusiform, 14.5-18 × 4-5.5 $\mu \mathrm{m}$. Appressoria in PDA slide cultures were brown to dark brown, subcircular to subfusoid, 4.5-10 × 4.5$8 \mu \mathrm{m}$ (Fig. 3). Teleomorph was not produced on media. These morphological characters were identical to Colletotrichum asianum (Prihastuti et al. 2009).

To confirm the morphological identification, the C. asianum isolates, PHP2017_Z23, PHP2017_Z25 and PHP2017_Z26,
Fig. 2 C. asianum PHP2017 Z23 growing on PDA plate after 10 days at $25^{\circ}$ C. (A) Upper plate showing acervuli around concentric rings (B) reverse side of the plate after 5 days at $25^{\circ} \mathrm{C}$

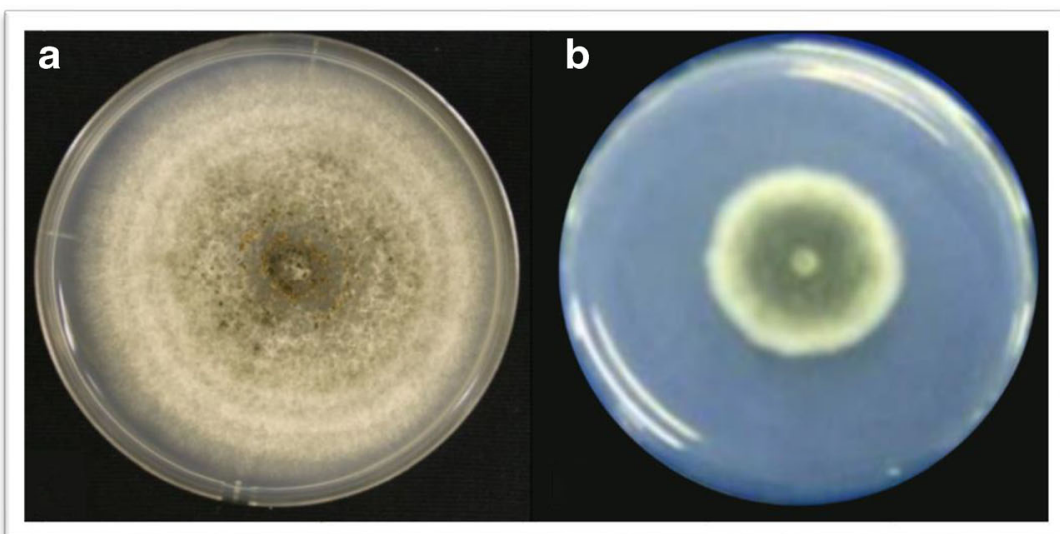


Fig. 3 Morphology and cultural characteristic of Colletotrichum asianum. (A) Conidia; (B and C) Appressoria; (D) conidial mass on plate culture

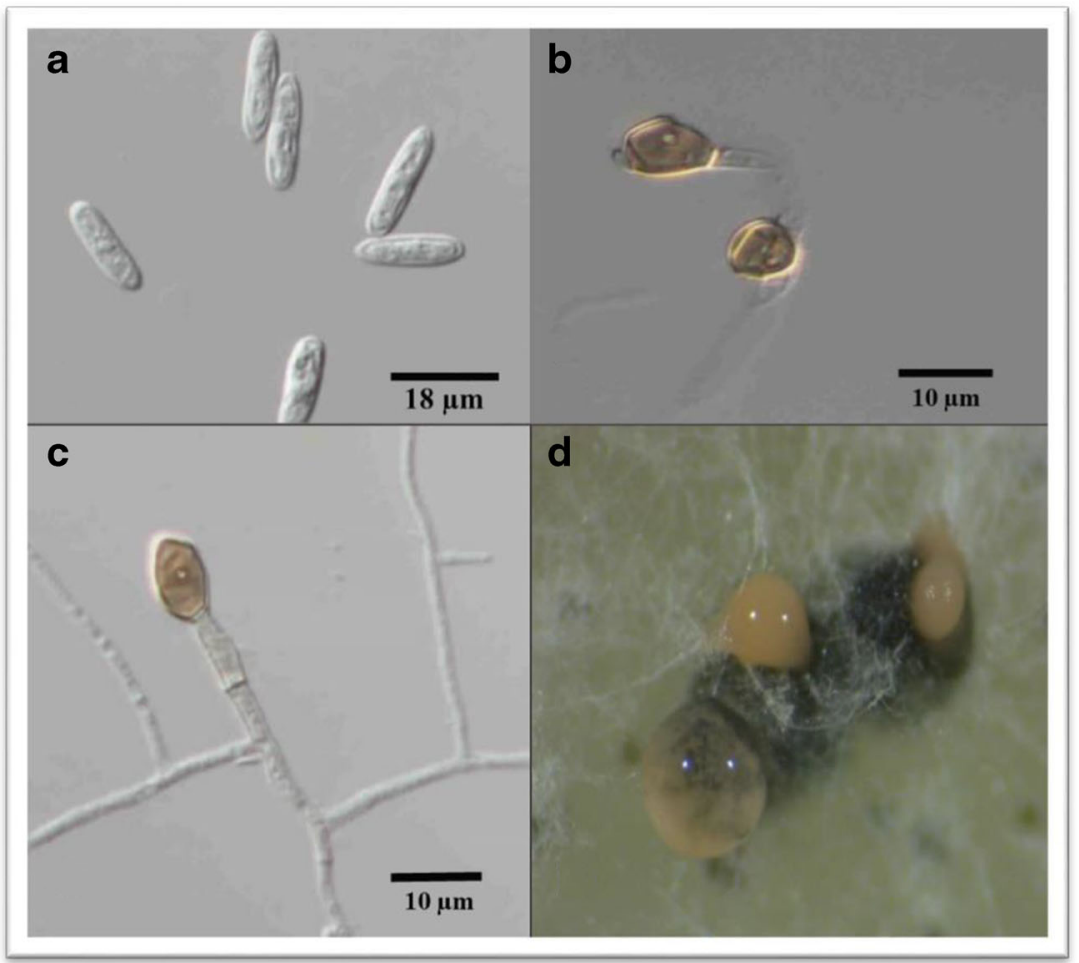

were subjected to amplification and sequencing of the internal transcribed spacer (ITS), actin (ACT), glyceraldehyde-3phosphate dehydrogenase (GAPDH), Calmodulin (CAL), and chitin synthase (CHS-1) gene regions utilizing primers V9G/ ITS4 (de Hoog and Gerrits van den Ende 1998; White et al. 1990), ACT512F/ ACT783R (Carbone and Kohn 1999), GDF/ GDR (Templeton et al. 1992), CL1-C/ CL2-C (Weir et al. 2012), and CHS-79F/ CHS-345R (Carbone and Kohn 1999), respectively. The NCBI GenBank Accession number for each gene (ITS, ACT, GAPDH, CAL and CHS) for each strain is LC458779, LC458783, LC458787 LC458791, LC458795 for PHP2017_23; LC458780, LC458784, LC458788, LC458792, LC458796 for PHP2017 25; and LC458781, LC458785, LC458789, LC458793 and LC458797 for PHP2017_26 respectively. The sequences were combined and aligned with sequences recollected from NCBI GenBank and were analyzed by Maximum parsimony (MP) using PAUP* v. 4.0 b 10 (Swofford 2002). Phylogenetic analyses showed that the isolates obtained from mango cv. Carabao were grouped in a same clade with an ex-type culture of Colletotrichum asianum (Fig. 5). This clade was strongly supported with $100 \%$ in Bootstrap value. From these results, isolates associated with anthracnose symptoms were identified as Colletotrichum asianum.

The isolates, PHP2017_Z23, PHP2017_Z25 and PHP2017 Z26, identified as Colletotrichum asianum were inoculated onto fruit of mango cv. Carabao to confirm their pathogenicity. Three closed containers each containing three healthy mango fruits, labeled A (unwounded), B (wounded by piercing the skin with disinfected needle) and C (control) were prepared

Fig. 4 Symptoms of anthracnose on fruit of mango cv. Carabao, seven days after inoculation with C. asianum PHP Z23; unwounded (A) and wounded (B) mango fruit $(\mathrm{C})$ control

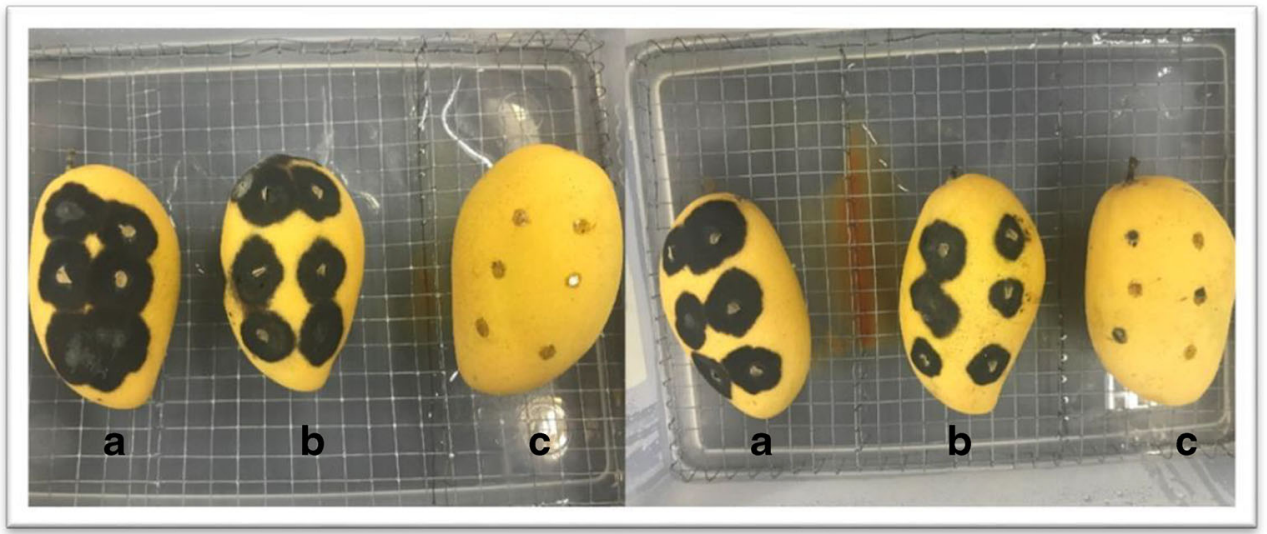


for each isolate. Six colonized agar blocks (10 $\mathrm{mm}$ in diameter) excised from seven-day old colonies of each isolate growing on PDA were placed on the surfaces of mango A and B (wounded area) while six blank agar blocks were placed on the surface of mango $\mathrm{C}$. The fruits were incubated for seven days under ambient light and temperatures $\left(25^{\circ}-30^{\circ} \mathrm{C}\right)$. Symptoms and signs of inoculated fruits were very similar to that of originally observed on the affected mangoes in the orchards (Fig.1; that is irregularly shaped, sunken lesions that later coalesced, with acervuli present in some of the infected fruits). The control fruits remained symptomless. The fungus was re-isolated from reproduced symptoms and the conidia exhibited the same morphological characters as the cultures used in the pathogenicity study (Fig. 4), thereby fulfilling the Koch's postulates.

Several reports on C. asianum associated with mango were reported among countries around the world, including

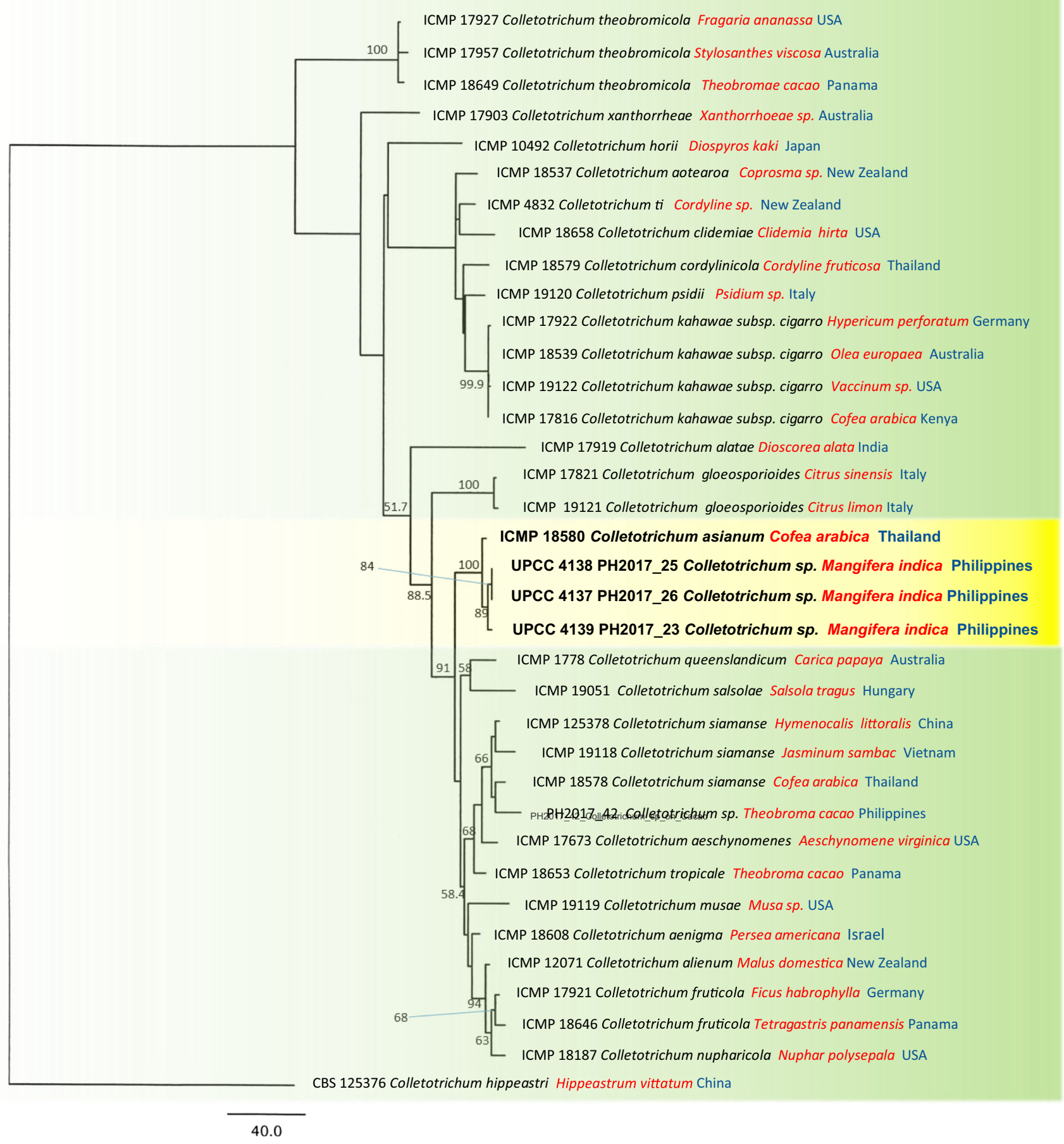

Fig. 5 Phylogenetic tree of Colletotrichum isolates grouped with ex-type Colletotrichum asianum strain 
Australia, Colombia, Japan and Panama (Weir et al. 2012). This paper reports Colletotrichum asianum as the causal fungus of anthracnose disease of mango cv. Carabao in Luzon and Guimaras Islands, Philippines using morphology, molecular analyses and pathogenicity testing. While a study published by Weir et al. (2012) mentioned C. asianum (MAFF306627) obtained from Philippine mango isolated from fruit imported into Japan, the isolate was used only to understand the species complex of $C$. gloeosporioides and did not formally introduced $C$. asianum as the causal fungi of anthracnose in Philippine mango cv. Carabao. Additionally, the pathogenicity of the Japanese isolate (MAFF306627) has not been confirmed (T. Sato. personal comm.).

Acknowledgements This study is carried out under the project designated by JSPS-DOST Joint Research Program and the Philippine Council for Agriculture, Aquatic and Natural Resources Research and Development of the Department of Science and Technology (DOST-PCAARRD). And also, this study is partially supported by Institute for fermentation, Osaka.

\section{References}

Alemu K (2014) Dynamics and Management of Major Postharvest Fungal Diseases of mango fruits. Journal of Biology, Agriculture and Healthcare 4:13-21

Alemu K, Ayalew A, Woldetsadic K (2014) Evaluation of antifungal activity of botanicals for postharvest management of mango anthracnose (Colletotrichum gloeosporioides). International Journal of Life Science 8(1):1-6

Alvindia DG, Acda MA (2015) Revisiting the efficacy of hot water treatment in managing anthracnose and stem-end rot diseases of mango cv. Carabao. Crop Prot 67:96-101

Carbone I, Kohn LM (1999) A method for designing primer sets for speciation studies in filamentous ascomycetes. Mycologia 91:553-556

Department of Agriculture-Bureau of Plant Industry (2016) High Value Crops Development Program- Mango Commodity Profile for March 2016. http://bpi.da.gov.ph/bpi/index.php/commodityprofile/186-mango-elements-paper-2016 Accessed 28 June 2019

Dodd JC, Estrada AB, Matcham J, Jeffries P, Jeger MJ (1991) The effect of climatic factors on Colletotrichum gloeosporioides, causal agent of mango anthracnose, in the Philippines. Plant Pathol 40:568-575. https://doi.org/10.1111/j.1365-3059.1991.tb02421.x

Esguerra EB, Chavez SM, Traya RV (2006) A modified and rapid heat treatment for the control of postharvest diseases of mango (Mangifera indica Linn. cv. Carabao) fruits. Philipp Agric Sci 89: 125-133

de Hoog GS, Gerrits van den Ende AHG (1998) Molecular diagnostics of clinical strains of filamentous Basidiomycetes. Mycoses 41:183189

Jeffries P, Dodd JC, Jeger MJ, Plumbley RA (1990) The biology and control of Colletotrichum species on tropical fruit crops. Plant Pathol 39:343-366

Lizada MCC, Agravante JU, Brown EO (1986) Factors affecting postharvest disease control in 'Carabao' mango subjected to hot water treatment. Philipp J Crop Sci 11:153-161

Nakashima C, Motohashi K, Chen C-Y, Groenewald JZ, Crous PW (2016) Species diversity of Pseudocercospora from Far East Asia. Mycol Prog 15:1093-1117

PSA (Philippine Statistics Authority) (2018) Major fruit crops quarterly bulletin 12(2), April-June Quezon City Philippines. ISSN 20946201. https://psa.gov.ph/content/major-vegetables-and-rootcropsquarterly-bulletin. Accessed 9 September 2019

Prakash O, Misra AK (2000) Diseases of fruits and vegetables and their management $1^{\text {st }}$ edition. Kalyani publishers pp 47-72

Prihastuti H, Cai L, Chen H, McKenzie EHC, Hyde KD (2009) Characterization of Colletotrichum species associated with coffee berries in northern Thailand. Fungal Divers 39:89-109

Simeon LM (2017) Department of Agriculture crafts 5-year roadmap for mango industry. The Philippine Star https://wwwphilstarcom/ business/2017/07/21/1720156/da-crafts-5-year-roadmap-mangoindustry Accessed 28 June 2019

Swofford DL (2002) PAUP*: Phylogenetic Analysis Using Parsimony (and other methods) 4.0b10. Sinauer, Sunderland, MA

Templeton MD, Rikkerink EHA, Solon SL, Crowhurst RN (1992) Cloning and molecular characterization of the glyceraldehyde-3phosphate dehydrogenase encoding gene and cDNA from the plant pathogenic fungus Glomerella cingulata. Gene 122:225-230

Weir BS, Johnston PR, Damm U (2012) The Colletotrichum gloeosporioides species complex. Stud Mycol 73:115-180. https:// doi.org/10.3114/sim0011

White TJ, Bruns T, Lee S, and Taylor JW (1990) Amplification and direct sequencing of fungal ribosomal RNA genes for phylogenetics. Pp. 315-322 In: PCR Protocols: A Guide to Methods and Applications, eds. Innis, M. A., D. H. Gelfand, J. J. Sninsky, and T. J. White. Academic Press, Inc., New York 